\title{
Leer y escribir como interpretación de roles, aprender de experiencias de estudiantes de doctorado
}

\author{
Writing and Reading as Role Enactment, \\ Learning through Ph. D. Students Experiences \\ Alma Cecilia Carrasco Altamirano \\ Benemérita Universidad Autónoma de Puebla \\ almacarrascoa@gmail.com \\ Margarita Flor de María Méndez Ochaita \\ Universidad Autónoma de Tlaxcala \\ flormendezo@yahoo.com \\ Rocio Brambila Limón \\ Universidad Autónoma de Tlaxcala \\ rocio.brambila76@gmail.com \\ María Teresa Fátima Encinas Prudencio \\ Benemérita Universidad Autónoma de Puebla \\ fatimaencinas@gmail.com \\ Verónica Sánchez Hernández \\ Benemérita Universidad Autónoma de Puebla \\ verher12@gmail.com
}

\begin{abstract}
3
Resumen

Este artículo reporta la investigación de las prácticas de escritura en dos programas de doctorado, en Fisiología y Oceanografía Física, para propiciar la construcción de vocaciones científicas desde el pregrado. Se optó por un paradigma cualitativo, utilizando entrevistas a profundidad con estudiantes, investigadores y administrativos, además de grupos focales con estudiantes. Para el análisis de los datos se utilizó el ATLAS.ti. Los resultados sugieren que los doctorandos se convierten en especialistas "en el trabajo" en un entorno colaborativo, supervisados y guiados por expertos. En este proceso formativo, los estudiantes juegan roles de autor, lector, crítico, editor y experto local al participar gradualmente en procesos de investigación, comités de política y gestión, paneles de revisión y comisiones de evaluación. Sobre todo, aprenden a escribir artículos, a revisarlos y a someterlos a dictaminaciones de comités científicos disciplinares.

Palabras clave: Aprendizaje situado, comunidades de práctica, literacidad disciplinaria.
\end{abstract}

32 - Leer y escribir como interpretación de roles, aprender de experiencias de estudiantes de doctorado Alma Carrasco, Margarita Méndez, Rocío Brambila, Ma. Teresa F. Encinas, Verónica Sánchez. Didac 75 (2020): $32-39$ 


\section{ABSTRACT}

This article presents the research of writing practices in two Ph.D. programs: Philosophy and Physical Oceanography, in order to promote the generation of scientific vocation from the undergraduate level. A qualitative approach was taken, based on depth interviews with students, researchers, and administrative staff, as well as focus groups with students. Atlas.ti was used to analyze the data. The results suggest that Ph.D. students become specialized at work through collaborative environments where they are supervised and guided by experts. During this training process, students play the role of author, reader, critic, editor, and local expert, all through gradually participating in research processes and on policy and management committees, review panels, and evaluation committees. First and foremost, they learn to write and review academic articles, then submit for review to scientific disciplinary committees.

Keywords: Situated learning, communities of practice, disciplinary literacy.

Fecha de recepción: Io/03/2019

Fecha de aceptación: I2/06/2019

\section{Introducción}

El número de programas de doctorado en México ha incrementado significativamente en las últimas décadas, como resultado de la política pública y el desarrollo endógeno de disciplinas y especialidades científicas. En 2016 el número de graduados de doctorado fue 8475 , el doble que hace diez años. De esta población de doctores, 850 se especializaron en ciencias naturales, ingeniería y ciencias biomédicas (Conacyt 229). Según el Science Report 2010: The Current Status of Science around the World de la Organización de las Naciones Unidas para la Educación, la Ciencia y la Cultura (Unesco 26), la formación científica y la productividad han aumentado desde el inicio del siglo Xxi a un ritmo más elevado en países menos desarrollados o de ingresos medios. Por lo tanto, existe un nuevo escenario para las ciencias en México.

A partir de 2010 se han incrementado los trabajos en la formación de doctores en México, particularmente en el ámbito de la escritura científica disciplinar (Ávila et al. 25; Castro y Sánchez 50-67; Espino 959-976; Trujeque et al.), pero se requieren estudios de experiencias de formación doctoral que aporten a la intervención educativa reflexiones sobre las exigencias en el posgrado que el pregrado puede empezar a vislumbrar.

Existe literatura reciente de distintas disciplinas sobre la formación científica en pregrado y posgrado. Un tema común es que la formación doctoral se reconoce como una trayectoria hacia una actividad de investigación que escasamente se realiza en licenciatura y que involucra los siguientes rasgos: un contexto físico, disciplinario y cultural específico; expectativas profesionales y desarrollo de la identidad; $y$ procesos hacia la enculturación y el desarrollo de la apropiación autoral a través de la producción de textos científicos en inglés (Bazerman 9-17; Campbell 897-927; Corcoran 242-255; Delamont y Atkinson 87-107; Englander 21-24; Englander y Corcoran 45-78; Mody y Kaiser 376- 402).

Por otro lado, Campbell (897-927), un antropólogo, plantea que el aprendizaje doctoral resulta de la supervisión formal y de procesos informales de enculturación que viven los estudiantes en la transición de ser estudiantes de pregrado a científicos, y que con confianza, competencia y acompañamiento son capaces de llevar a cabo investigación científica desde la construcción del marco conceptual hasta la publicación de artículos.

De acuerdo con un punto de vista sociológico, Laudel y Gläser (387-406) caracterizan el desarrollo del estudiante como un cambio de estado, de aprendiz a colega, que comprende tres dimensiones: cognitiva, comunitaria y organizacional. Prior (110-125) concibe la escritura disciplinaria como formas duraderas de alineación y perspectiva a través del tiempo, lo cual conforma trayectorias de participación en prácticas disciplinarias.

Desde la perspectiva de la investigación educativa, Bloomer y Hodkinson (583-597) conceptualizan 
esta trayectoria como carrera de aprendizaje, mientras que, según el punto de vista de las comunidades de práctica, puede considerarse como "un aprendizaje cognitivo [en] una variedad de situaciones, incluyendo instituciones educativas, ámbitos de trabajo y entornos informales de participación" (584). De algún modo, las instituciones de educación superior podrían contribuir a estas carreras de aprendizaje científico.

En el ámbito de la actividad científica, la trayectoria formativa no siempre ocurre de forma lineal. Es importante reconocer que el tránsito de novato a experto exige la participación continua del aprendiz de científico en distintas actividades y de la adopción de diversos roles que posibiliten cumplir con las tareas que toda investigación exige.

Este trabajo da cuenta de la participación de estudiantes doctorales en proyectos de investigación, con distintos grados de autonomía, a través de la adopción de varios roles. Se busca derivar lecciones para apoyar la formación disciplinar desde el pregrado al reconocer los diferentes roles llevados a cabo por los científicos en su trabajo.

\section{Marco de referencia y objetivos de la investigación}

Este artículo se sustenta en las tradiciones mencionadas anteriormente y presenta tres distinciones generales para investigar las trayectorias de formación de científicos: 1) la experiencia formativa que brinda la organización social de la institución (Laudel y Gläser 387-406); 2) los modos culturales y las diferentes prácticas comunicativas en contextos organizacionales y disciplinarios específicos (Becher y Trowler 40-57); y 3) el proceso de producción escrita (Bazerman 9-17; Overington 143-164; Prelli 87-111; Prior 110-125).

También se presentan conexiones entre los procesos de escritura y los diversos roles asumidos por los científicos y, presumiblemente, por sus estudiantes como participantes en comunidades de práctica que desarrollan aprendizajes periféricos legitimos (Lave y Wenger 63-82).

A menudo se olvida que un científico consolidado no es simplemente un investigador que realiza su actividad científica y publica artículos; en realidad, realiza muchos otros quehaceres (Bazerman 9-17). Continuamente prepara propuestas de investigación, busca financiamiento, revisa artículos para revistas, participa en comisiones que dictaminan propuestas para asignar financiamiento, organiza eventos académicos, coordina trabajos de estudiantes y asistentes de investigación, da conferencias, supervisa tesis, dirige experimentos, supervisa la instalación y mantenimiento de equipos, mantiene comunicación con colegas en otras universidades, atrae a profesores visitantes a su centro, prepara informes para la administración y participa en el diseño de políticas institucionales y en actividades de gestión. La actividad científica es compleja, múltiple y exige la realización de diversos papeles. Bazerman (146) sostiene que:

todos los roles para la comunicación como colaborador, revisor, crítico y lector pueden ser realizados en distintos momentos por cada investigador [...] cada rol literalmente toma el lugar del otro [...] el científico investigador aprende a entender, aceptar y conocer las expectativas y demandas de la audiencia.

Se reconocen tres dimensiones de los roles: patrones de comportamiento social, identidades y guiones o expectativas de comportamientos asumidos por otros actores (Jackson 49-55). Al identificarse con un rol y asumir la responsabilidad de éste, los científicos progresivamente incorporan un habitus (Bourdieu 84-104). Los doctorandos aprenden poco a poco a adoptar diversos roles a medida que asisten a sus asesores en una serie de tareas, las cuales van más allá del aprendizaje formal en seminarios o de la redacción de experimentos o de la realización de trabajo de campo para su proyecto de tesis. El campo científico en el que operan es una estructura social compleja que exige realizar diversas actividades que representan patrones y símbolos alrededor de los cuales los participantes desempeñan múltiples roles.

Los roles desempeńados por estudiantes e investigadores son claves para la reproducción social de las estructuras institucionales y culturales de la ciencia. Desde la perspectiva individual, los roles se promulgan y tienen sentido -son legitimados- en el contexto de las instituciones. Esta socialización científica tiene múltiples dimensiones que pueden explicarse desde tres perspectivas complementarias: 1) la investigación sociológica (Laudel y Gläser 387-

34 - Leer y escribir como interpretación de roles, aprender de experiencias de estudiantes de doctorado Alma Carrasco, Margarita Méndez, Rocío Brambila, Ma. Teresa F. Encinas, Verónica Sánchez. Didac 75 (2020): 32-39 
406), 2) los estudios antropológicos (Delamont y Atkinson 87-107) y 3) la investigación de literacidad social (Prior 110-125). Los aprendices de investigación son socializados en:

- Una dimensión institucional al experimentar la ciencia como una profesión (Merton 267-278).

- Una comunidad disciplinaria al validar las formas de participación y reconocimiento en conferencias, revistas y redes colaborativas.

- Una ruta de investigación práctica y cognitiva marcada por las exigencias disciplinarias. Esto implica la realización de ciertos trabajos de investigación (ejecutar procedimientos, recopilar datos de campo, producir resultados, redactar un artículo de investigación).

Por lo tanto, un científico lleva a cabo muchos tipos de actividades y desempeña diferentes roles en varias esferas como: a) miembro de la profesión científica, b) participante en su comunidad disciplinaria y c) profesional de la producción de conocimiento. Convertirse en un científico que se desempeña profesionalmente en una especialidad disciplinaria conduce a un aprendiz a producir publicaciones revisadas por pares, lo que implica múltiples desafíos. Mucho antes de que los estudiantes se conviertan en autores ya observan cómo estos desafíos se expresan en actividades, roles y prácticas de escritura de sus compañeros más avanzados y de sus profesores.

Resulta útil examinar experiencias del doctorado de aprendices que participan en actividades científicas institucionalizadas adoptando roles legítimos en tres dimensiones: profesional, comunitaria y práctica-cognoscitiva. Delamont y Atkinson (87107) señalan que la mayoría de estas actividades no están formalmente codificadas o "enseñadas" sino "aprendidas", ya que son típicamente habilidades y formas de conocimiento tácitos que de forma progresiva se incorporan como habitus.

Este estudio exploratorio adopta la postura de Bazerman (9-17) y se pregunta si la transformación de principiante a experto puede entenderse parcialmente como la puesta en marcha sucesiva de roles científicos específicos. Para responder al planteamiento, partimos de la identificación de los siguientes roles: autor, lector, crítico, editor y experto local (Campbell 897-927).

\section{Método}

El enfoque metodológico es cualitativo. En el marco de un proyecto de investigación iniciado en 2010 sobre la formación de científicos se entrevistó a jefes de laboratorios, investigadores y doctorandos de distintos centros y disciplinas: biotecnología, astrofísica, agricultura, fisiología y oceanografía física. Las entrevistas duraron 45 minutos en promedio. En dos de estos centros se organizaron grupos focales con estudiantes. Todas las entrevistas fueron grabadas y transcritas a Word. Luego se analizaron utilizando ATLAS.ti.

En el presente trabajo se emplea el material empírico de fisiólogos y oceanógrafos recolectado en mayo de 2011. Estos datos ejemplifican el ejercicio de roles en la formación de doctores de dos disciplinas: fisiología y oceanografía física.

\section{Contexto de estudio}

El Instituto de Fisiología se estableció en 1991 en la Benemérita Universidad Autónoma de Puebla (BUAP). Ofrece un programa de maestría -creado en 1984-y otro de doctorado-1994-, y participa en un programa de licenciatura en Ciencias Biomédicas en colaboración con la Facultad de Medicina de la Universidad de Puebla (véase <http://www.fisio.BUAP.mx>).

El doctorado en Oceanografía Física es ofrecido por el departamento del mismo nombre en el Centro de Investigación Científica y Educación Superior (CICESE) en Ensenada, Baja California, en el noroeste de México. Este instituto público de investigación fue fundado en 1973 por el gobierno federal como un medio para diversificar y descentralizar la actividad científica en México y se ha convertido en uno de los principales centros de investigación a nivel nacional. Tiene una amplia colaboración con la industria, la agricultura y los sistemas nacionales de salud, geología y meteorología.

La investigación en fisiología se lleva a cabo en el laboratorio, donde investigadores, estudiantes -de licenciatura, maestría y doctorado-, profesores de 
posdoctorado y visitantes interactúan continuamente. La colaboración adopta varias formas durante los procedimientos experimentales, con el propósito de llevar a cabo el análisis de datos y la producción escrita.

Mientras que en el CICESE las actividades se organizan de manera diferente, pues los oceanógrafos deben recopilar datos y muestras sobre viajes oceánicos realizados una vez al año. Cabe puntualizar que el investigador principal que dirige un viaje puede o no ser el director de tesis del estudiante. El trabajo de campo es una actividad compleja e intensa que involucra la preparación de un viaje, la toma de datos, la recolección de muestras y la interacción con personas que trabajan en otras especialidades. Este tipo de actividad es a menudo menos controlada que los procedimientos de investigación en un laboratorio. En este proceso, las rutinas se ejecutan individualmente y la colaboración es menos espontánea que en un laboratorio. Por lo tanto, la iniciativa estudiantil está detrás de muchas de las interacciones cruciales con compañeros y profesores.

A pesar de las diferencias en la organización formativa de los dos doctorados, los estudiantes de ambos programas trabajan con datos, escriben, participan en seminarios de investigación y representan distintos roles.

\section{Participantes}

Se entrevistó a seis investigadores y a 12 estudiantes de doctorado (ver Tabla 1).

\section{TABla I. Características de los participantes}

\begin{tabular}{ccc} 
Participantes & Mujer & Hombre \\
\hline Estudiantes & 5 & 7 \\
\hline Investigadores & I & 5 \\
\hline
\end{tabular}

Nota: Para la clave de registro de entrevistas:

Fisiología: FISIOB

Oceanografía: OCEAN

Estudiantes: $\mathrm{E}$

Investigadores: $\mathrm{H}$

Mujeres: A

Hombres: o

Los sujetos de estudio son hablantes nativos de español que publican artículos de investigación en inglés.

\section{Resultados y discusión}

En estos contextos, el tutor guía el desarrollo progresivo de los estudiantes hacia prácticas de escritura que se utilizan en la comunidad disciplinar. Éstas llevan al tutor y al estudiante a representar una variedad de roles. La presente sección expone fragmentos de entrevistas para ejemplificarlos.

En su rol de autor, el estudiante se esfuerza por apropiarse del texto como su producto, independientemente de si éste fue producido con la ayuda de otros pares o del tutor. Un estudiante avanzado afirma que ser autor es convertirse en un experto que comunica y ensancha la frontera del conocimiento:

FISIOB EO7: Aprendes mucho del oficio del científico, especialmente publicando, divulgando tus hallazgos del laboratorio [...] transmitirlos a los estudiantes de maestría y licenciatura más jóvenes [...] enseñarles el quehacer científico, dar a conocer y discutir los resultados en la comunidad científica, en congresos, por ejemplo.

Aprender a ser autor también significa hacer pequeñas contribuciones al artículo a lo largo del proceso de producción:

FISIOB EO4: Yo no redacté todo el artículo, sólo aportaba ciertas cosas en cuanto en el texto y las figuras, sí, tuve más participación en la creación de las figuras y notas al pie de figura también.

Convertirse en autor implica reconocer y desarrollar un trabajo importante realizado por otras personas en la producción de datos. La autoría es colaborativa:

OCEAN EA1: Trabajo con una base de datos [...]. Éstos son los datos obtenidos por otros estudiantes en sus viajes. Ahora los utilizo y contribuyo. Los datos que colecciono en mis viajes son cosas que usaré, pero también serán utilizados por otros.

Asimismo, los estudiantes están conscientes de la oportunidad de contribuir al conocimiento en su disciplina, pero enfrentan desafíos para cumplir con las normas de los modos de expresión del inglés científico:

36 - Leer y escribir como interpretación de roles, aprender de experiencias de estudiantes de doctorado Alma Carrasco, Margarita Méndez, Rocío Brambila, Ma. Teresa F. Encinas, Verónica Sánchez. Didac 75 (2020): 32-39 
ocean ea4: No soy autosuficiente. Proporcioné la estructura básica [de una buena idea] pero él [el profesor] hace la edición.

Finalmente, aprender a ser autor también requiere instruirse en examinar los artículos desde la perspectiva de un editor. Es decir, exige conocer los criterios editoriales de las revistas científicas para presentar contribuciones y hacer revisiones sugeridas por el tutor:

FISIO HO3: Las dos revistas en las que preferimos publicar, el Journal of Neuroscience y el Journal of Neurophysiology, son el estándar que establecí para mis estudiantes. Esto es lo que aspiramos, publicar en estas revistas y otras de alto impacto. El estudiante debe tener esto en cuenta: queremos alcanzar esos niveles, alcanzar esos estándares [...] Así es como motivas a los estudiantes.

Los oceanógrafos de este estudio leen $-\mathrm{y}$ tratan de publicar en- Fluid Mechanics y Journal of Physical Research:

OCEAN EA1: A lo mejor todo el tiempo te dedicaste a estudiar mecánica de fluidos, pero para escribir, redactar y publicar necesitas saber de otras cosas: redacción de estilo, gramática y un montón de otras cosas.

Reconocer el impacto de las revistas en la comunidad académica es aprender sobre cómo funciona el mundo de los editores de revistas. Por lo tanto, el autor que envía un artículo es el primer crítico de su propio trabajo. La crítica editorial se internaliza, ya que es parte de aprender a ser un científico que, al mismo tiempo, se inicia en las prácticas de la comunidad disciplinaria específica.

Esto también implica saber cómo funciona la revisión por pares en la práctica. Los estudiantes aprenden que los revisores no juzgan los artículos que dictaminan con los mismos estándares:

FISIO HO11: Lo primero que hago es mandar un artículo a una revista muy reconocida en Estados Unidos, si la aceptan bien. Pero si no la aceptan es generalmente por asuntos menores, una especie de racismo intelectual es más común en Estados Unidos. Entonces, lo mando a una revista europea y es más fácil que te publiquen ahí porque Europa es pluricultural. No les importa mucho de dónde es uno. He encontrado editores que preguntan dónde está Puebla sin leer el artículo. En otra buena revista es difícil que te publiquen sin una recomendación. Es como una pequeña mafia editorial.

Cuando los aprendices de científicos citan a los autores que consideran valiosos para su trabajo actúan en el rol de crítico y logran dos requisitos de la actividad científica: reconocer las contribuciones de otros investigadores y cultivar su "pertenencia" a una comunidad de especialistas que trabajan una misma línea de investigación.

La consecuencia natural de convertirse en un experto es reconocer que se compite con otras personas que trabajan temas similares. El que llega primero toma el crédito. Aprender a ser un crítico es asimilar las prácticas y los criterios -a menudo-implícitos de la institución científica.

A veces en el laboratorio ciertas personas se hacen competentes en el manejo de datos o el uso de un determinado tipo de equipo. Campbell (897-927) llama a estos individuos expertos locales y en este trabajo se identifica que pueden ser estudiantes o académicos del mismo centro o de otros, y los demás investigadores suelen recurrir a ellos para resolver asuntos que son de su experticia:

OCEAN EA1: A veces tienes un problema-como cómo resolver una ecuación- y luego son tus compañeros estudiantes que podrían ayudar. Incluso podría ser el autor de un artículo que leíste -le envías un e-mail y le pides ayuda-. Eso es una cosa que me gusta de esto, pertenecer a una comunidad.

Lave y Wenger plantean que "las comunidades de práctica tienen historias y ciclos de desarrollo y se reproducen de tal manera que la transformación de los recién llegados en veteranos se vuelve poco notable e integral a la práctica" (122). Aprender a ser científico no es el resultado de la enseñanza explícita; más bien, es la consecuencia de actuar como científico acompañado de otros que adoptan distintos roles. 


\section{Conclusiones}

Como parte de sus rutinas, un científico experimentado lleva a cabo una variedad de actividades en diversas esferas institucionales. En el ámbito práctico-cognitivo, realiza procedimientos concretos en un área específica de conocimiento; en el ámbito profesional, participa en comités de política y gestión, paneles de revisión y comisiones de evaluación; como miembro de una comunidad disciplinaria, revisa artículos sometidos a las revistas y protocolos de investigación y trabaja en comités científicos de congresos.

Cuando los profesores invitan a sus estudiantes de doctorado a ayudar en estas tareas, se crea un mecanismo de socialización en el que los estudiantes son iniciados en la ciencia como profesión, como actores con una identidad disciplinaria, y expresan una práctica cognitiva en la ejecución de procedimientos específicos de recolección de datos, análisis, instrumentación y escritura. Al observar a los expertos actuando en diversos roles, los aprendices se inician en la comprensión de las reglas del juego de la ciencia.

Los estudiantes aprenden la escritura en estas comunidades disciplinares, fisiología y oceanografía, con un acompañamiento continuo, tanto de expertos como de otros estudiantes de licenciatura, maestría o doctorado. Esto sugiere repensar "la enseñanza" de la escritura y enfatizar más su aprendizaje desde el pregrado. También lleva a reflexionar sobre la necesidad de crear espacios en las universidades, como centros de escritura, donde los estudiantes puedan elaborar proyectos propios de cada disciplina con el acompañamiento de expertos y colegas del mismo programa.

En el rol de aprendiz se observa que el estudiante concibe la actividad científica como un proceso emergente al desarrollar habilidades y conocimientos tácitos. Los estudiantes devienen como especialistas en el trabajo en un entorno colaborativo altamente valorado en las disciplinas estudiadas de las ciencias naturales y exactas.

Reconocer el ejercicio de los roles de autor, editor, crítico y experto local-sin dejar de considerar las diferencias disciplinarias- podría constituir un recurso formativo para orientar vocaciones científicas desde el pregrado. En el doctorado de Fisiología, estudiantes y profesores interactúan de forma continua en un entorno físico cerrado, se percibe que aprender a ejecutar procedimientos de laboratorio y escribir distintos textos que alimentan la publicación se relaciona con la representación de estos roles. En cuanto a los oceanógrafos, hay experiencias similares de emergencia y apropiación de roles. Además, se identifica la existencia de redes de actores que se extienden más allá del entorno local e inmediato de investigación. Los expertos locales y los externos actúan como tutores o acompañantes formativos de los recién llegados.

Otro aspecto que surge de la comparación de estas disciplinas se refiere a las formas específicas de la organización social estudiantil. En un laboratorio de fisiología, la interacción en el grupo es una necesidad física y de procedimiento, mientras que no es así con los estudiantes del doctorado en Oceanografía, pues en su labor cotidiana tienden a trabajar individualmente. Estudiantes de ambos programas se esfuerzan para organizar las interacciones con sus pares y desarrollar seminarios independientes de formación sin la guía de sus profesores (fuera de las exigencias y regulaciones institucionales).

En materia de escritura científica, las similitudes de los retos reconocidos por los aprendices son evidentes. Es probable que éstas resulten de la estandarización global de la publicación científica que, en las disciplinas estudiadas, se realiza en inglés.

Aprender inglés científico implica una transición cultural que no es analizada a profundidad en este artículo. En el caso mexicano, se podría decir que los doctorandos de Fisiología y Oceanografía Física transitan de un sistema educativo monolingüe a un sistema científico bilingüe. Es necesario investigar las implicaciones de estas transiciones culturales y lingüísticas entre los sistemas educativos nacionales y el inglés científico global.

Para obtener una comprensión comparativa más clara del aprendizaje de roles y las representaciones de éstos en diferentes disciplinas sería necesario observar a los investigadores llevar a cabo otras actividades, como el trabajo de campo, la planificación, las conferencias y la gestión. I

38 - Leer y escribir como interpretación de roles, aprender de experiencias de estudiantes de doctorado Alma Carrasco, Margarita Méndez, Rocío Brambila, Ma. Teresa F. Encinas, Verónica Sánchez. Didac 75 (2020): 32-39 
Ávila, Alicia, et al., coords. Una década de investigación educativa en conocimientos disciplinares en México: matemáticas, ciencias naturales, lenguaje y lenguas extranjeras: 2002-2011. México: ANuies/Consejo Mexicano de Investigación Educativa, 2013. Impreso.

Bazerman, Charles. Shaping Written Knowledge: The Genre and Activity of the Experimental Article in Science. Madison: University of Wisconsin Press, 1988. Impreso.

Becher, Tony, y Paul R. Trowler. Academic Tribes and Territories: Intellectual Enquiry and the Culture of Disciplines. Buckingham: Society for Research into Higher Education/Open University Press, 2001. Impreso.

Bloomer, Martin, y Phil Hodkinson. "Learning Careers: Continuity and Change in Young People's Dispositions to Learning." British Educational Research Journal 26, 5 (2000): 583-597. Impreso.

Bourdieu, Pierre. "Le Champ Scientifique." Actes de la Recherche en Sciences Sociales 2, 2 (1976): 84-104. Impreso.

Campbell, Robert A. "Preparing the Next Generation of Scientists: The Social Process of Managing Students." Social Studies of Science 33, 6 (2003): 897-927. Impreso.

Castro, María Cristina, y Martín Sánchez. "Escribir en la universidad: la organización retórica del género tesina en el área de humanidades." Perfiles Educativos 37, 148 (2015): 50-67. Impreso.

Consejo Nacional de Ciencia y Tecnología (Conacyt). Informe General del Estado de la Ciencia y Tecnología. México: Conacyt, 2016. Impreso.

Corcoran, James. "The Potential and Limitations of an English for Research Publication Purposes Course for Mexican Scholars." Global Academic Publishing Policies, Perspectives and Pedagogies. Ed. Mary Jane Curry y Theresa Lillis. Bristol: Multilingual Matters, 2017. 233-248. Impreso.

Delamont, Sara, y Pauln Atkinson. "Doctoring Uncertainty: Mastering Craft Knowledge.” Social Studies of Science 31, 1 (2001): 87-107. Impreso.

Englander, Karen. Writing and Publishing Science Research Papers in English: A Global Perspective. Holanda: Springer, 2013. Impreso.
Englander, Karen, y James Corcoran. English for Research Publication Purposes: Critical Plurilingual Pedagogies. Oxon/ Nueva York: Taylor \& Francis, 2019. Impreso.

Espino, Sandra. "La enseñanza de estrategias de escritura y comunicación de textos científicos y académicos a estudiantes de posgrado." Revista Mexicana de Investigación Educativa 20, 66 (2015): 959-976. Impreso.

Jackson, Jeanne. "Contemporary Criticisms of Role Theory." Journal of Occupational Science 5, 2 (1998): 49-55. Impreso.

Laudel, Grit, y Jochen Gläser. "From Apprentice to Colleague: The Metamorphosis of Early Career Researchers." Higher Education 55, 3 (2008): 387-406. Impreso.

Lave, Jean, y Etienne Wenger. Situated Learning: Legitimate Peripheral Participation. Cambridge: Cambridge University Press, 2011. Impreso.

Merton, Robert K. "The Normative Structure of Science.” The Sociology of Science: Theoretical and Empirical Investigations. Ed. Robert K. Merton. Chicago/Londres: University of Chicago Press, 1973. Impreso.

Mody, Cyrus C. M., y David Kaiser. "Scientific Training and the Creation of Scientific Knowledge." The Handbook of Science and Technology Studies. Eds. Edward Hackett et al. Cambridge/Londres: The мIт Press, 2008. 377- 402. Impreso.

Organización de las Naciones Unidas para la Educación, la Ciencia y la Cultura (Unesco). Unesco Science Report 2010: The Current Status of Science around the World. París: Unesco, 2010. Impreso.

Overington, Michael A. "The Scientific Community as Audience: Toward a Rhetorical Analysis of Science.” Philosophy \& Rhetoric 10, 3 (1977): 143-164. Impreso.

Prelli, Lawrence J. A Rhetoric of Science: Inventing Scientific Discourse. Madison: University of South Carolina Press. 1989. Impreso.

Prior, Paul A. Writing/Disciplinarity: A Sociohistoric Account of Literate Activity in the Academy. Mahwah, Nueva Jersey: Lawrence Erlbaum Associates, 1998. Impreso.

Trujeque, Eva Estefania, et al. "Exploring Authorship Development among Mexican EfL Teacher-Researchers." Profile Issues in Teachers' Professional Development 17, 2 (2015): 43-62.

\section{Semblanzas}

Alma Cecilia Carrasco Altamirano. Académica de la BUAP y Presidenta del Consejo Puebla de Lectura A.C. Sus líneas de investigación son: fomento a la lectura y bibliotecas; leer en primera infancia; trayectorias formativas de científicos y autoría y, lenguaje y currículo.

Margarita Flor de María Méndez Ochaita. Doctora en Ciencias en la especialidad de Investigaciones Educativas por el Cinvestav-IPN. Profesora en la Maestría en Estudios del Discurso y Literacidades Académicas de la Universidad Autónoma de Tlaxcala.

Rocío Brambila Limón. Estudiante del doctorado en Educación en la Universidad Autónoma de Tlaxcala y maestra en Administración y Gestión de Instituciones Educativas por la BUAP. Sus líneas de investigación son: literacidad e investigación y, trayectorias de desarrollo profesional y autoria.

María Teresa Fátima Encinas Prudencio. Profesora en la Maestría y Licenciatura en Ensañanza del Inglés de la Facultad de Lenguas de la BUAP. Sus líneas de investigación son: literacidad e investigación y, trayectorias de desarrollo profesional y autoria.

Verónica Sánchez Hernández. Doctora en Lingüística Aplicada por la Universidad de Macquarie, Australia. Es docente-investigadora en la Facultad de Lenguas de la BUAP. Sus líneas de investigación son: formación docente; desarrollo profesional; supervisión; desarrollo de literacidad y procesos de acreditación. 